\title{
Analysis of Vibration Influencing Factors of High Precision Cylindrical Roller Bearing by Poor Information (Part II : Experiment)
}

\author{
Xintao Xia ${ }^{1, a}$, Bin Liu ${ }^{1, b^{*}}$, Xiaowei Yang ${ }^{2, c}$ and Wunhuan Zhu ${ }^{1, d}$ \\ ${ }^{1}$ Mechatronical Engineering College, Henan University of Science and Technology, Luoyang \\ 471003, China \\ ${ }^{2}$ Luoyang Bearing Science \& Technology Co., Ltd., Luoyang 471039, China \\ aemail:xiaxt1957@163.com, bemail:Ibin1992@foxmail.com, cemail:yangxwzys@163.com, \\ demail:zhuwenhuan0916@126.com \\ * The Corresponding author
}

Keywords: High precision cylindrical roller bearing; Vibration velocity; Correlation sequence; Factor analysis

\begin{abstract}
The vibration of high precision cylindrical roller bearings is affected by many factors, which belongs to the problem of poor information. The experimental results show that the main factors influencing the vibration of the experimental bearing are the clearance, the roller convexity and roughness, the inner ring waviness and roughness, and the inner and outer ring flange roughness.
\end{abstract}

\section{Introduction}

High precision cylindrical roller bearings as precision mechanical components, have higher quality requirement than normal ones. As an important connection basic part of mechanical, the rolling bearing's quality is very significant and plays a very important role in the normal operation of the equipment. Many studies have been conducted on their quality [1-7]. Literatures [1-3] studied the bearing quality from the aspects of material, load, life. Literatures [4-7] used different methods to analyze all aspects of the bearing.

According to the poor information system theory $[8,9]$, a variety of mathematical methods are used, and then those obtained results are compared and analyzed according to the qualitative fusion principle [10] to obtain a common solution. This result is the major factor influencing the vibration velocity of the experimental bearing, namely the final solution of the problem.

\section{Analysis of Influencing Factors}

The research object of this experiment is the vibration problem of NJ203 type high precision cylindrical roller bearing. Through the experiment measurement, the parameters of the inner and outer rings and the roller are obtained, and the relationship between the parameters and the bearing vibration is studied by different methods. Qualitative fusion is used to find out the main influencing factors.

The 19 ring parameters, 16 roller parameters and 1 overall parameter of the bearing were selected as the research objects, and the ring parameters included 9 inner ring parameters and 10 outer ring parameters. The relationship between the parameters of the bearing and the vibration velocity of the bearing is studied by experiment.

In order to facilitate the study, the symbols and meanings used in the experimental study are shown in Table 1. 
Table 1 Symbols and meanings

\begin{tabular}{cclccc}
\hline Symbol & Meaning & Component & Symbol & Meaning & Component \\
\hline$X_{1}$ & $S_{\mathrm{d}}$ & inner race & $X_{20}$ & $D_{\mathrm{w}}(\mathrm{A})$ & roller \\
$X_{2}$ & $K_{\mathrm{i}}$ & inner race & $X_{21}$ & $\Delta L W s(\mathrm{~A})$ & roller \\
$X_{3}$ & $S_{\mathrm{di}}$ & inner race & $X_{22}$ & bases runout (A) & roller \\
$X_{4}$ & $S_{\mathrm{if}}($ flange) & inner race & $X_{23}$ & convexity (A) & roller \\
$X_{5}$ & $L_{\mathrm{i}}$ & inner race & $X_{24}$ & roundness (A) & roller \\
$X_{6}$ & roundness & inner race & $X_{25}$ & waviness (A) & roller \\
$X_{7}$ & waviness & inner race & $X_{26}$ & roughness (A) & roller \\
$X_{8}$ & roughness & inner race & $X_{27}$ & bases roughness(A) & roller \\
$X_{9}$ & roughness(flange) & inner race & $X_{28}$ & $D_{\mathrm{w}}(\mathrm{D})$ & roller \\
$X_{10}$ & $S_{\mathrm{D}}$ & outer race & $X_{29}$ & $\Delta L W s$ (D) & roller \\
$X_{11}$ & $K_{\mathrm{e}}$ & outer race & $X_{30}$ & bases runout (D) & roller \\
$X_{12}$ & $S_{\mathrm{e}}$ & outer race & $X_{31}$ & convexity (D) & roller \\
$X_{13}$ & $S_{\mathrm{ef}}$ (flange) & outer race & $X_{32}$ & roundness (D) & roller \\
$X_{14}$ & raceway width & outer race & $X_{33}$ & waviness (D) & roller \\
$X_{15}$ & $L_{\mathrm{e}}$ & outer race & $X_{34}$ & bases roughness(D) & roller \\
$X_{16}$ & roundness & outer race & $X_{35}$ & bases roughness(D) & roller \\
$X_{17}$ & waviness & outer race & $X_{36}$ & clearance & overall \\
$X_{18}$ & roughness & outer race & $Y_{1}$ & Low frequency vibration velocity \\
$X_{19}$ & roughness(flange) & outer race & $Y_{2}$ & Intermediate frequency vibration velocity \\
& & & $Y_{3}$ & High frequency vibration velocity \\
\hline
\end{tabular}

In Table 1, "A" represents the average of 11 rolling elements in a set of bearings, and "D" represents the difference between the 11 rolling elements in a set of bearings

The bearing vibration velocity of each frequency band is shown in Table 2

Table 2 The bearing vibration velocity of each frequency band $[\mu \mathrm{m} / \mathrm{s}]$

\begin{tabular}{cccccccccccccccc}
\hline Number & 1 & 2 & 3 & 4 & 5 & 6 & 7 & 8 & 9 & 10 & 11 & 12 & 13 & 14 & 15 \\
\hline$Y_{1}$ & 390 & 150 & 240 & 120 & 320 & 160 & 260 & 150 & 220 & 270 & 120 & 300 & 300 & 300 & 260 \\
$Y_{2}$ & 400 & 280 & 240 & 170 & 320 & 260 & 220 & 260 & 410 & 180 & 260 & 240 & 320 & 180 & 300 \\
$Y_{3}$ & 170 & 120 & 180 & 150 & 150 & 140 & 160 & 180 & 240 & 90 & 180 & 190 & 140 & 130 & 140 \\
\hline \multicolumn{10}{c}{ Continued table 2} \\
\hline Number & 16 & 17 & 18 & 19 & 20 & 21 & 22 & 23 & 24 & 25 & 26 & 27 & 28 & 29 & 30 \\
\hline$Y_{1}$ & 200 & 220 & 150 & 240 & 220 & 180 & 330 & 240 & 150 & 270 & 420 & 210 & 270 & 300 & 140 \\
$Y_{2}$ & 210 & 290 & 170 & 150 & 270 & 180 & 240 & 380 & 170 & 300 & 320 & 240 & 300 & 310 & 230 \\
$Y_{3}$ & 140 & 130 & 150 & 150 & 140 & 180 & 150 & 120 & 130 & 150 & 140 & 120 & 110 & 140 & 170 \\
\hline
\end{tabular}

The remaining parameters affecting the measurement data are shown in Fig. 1 


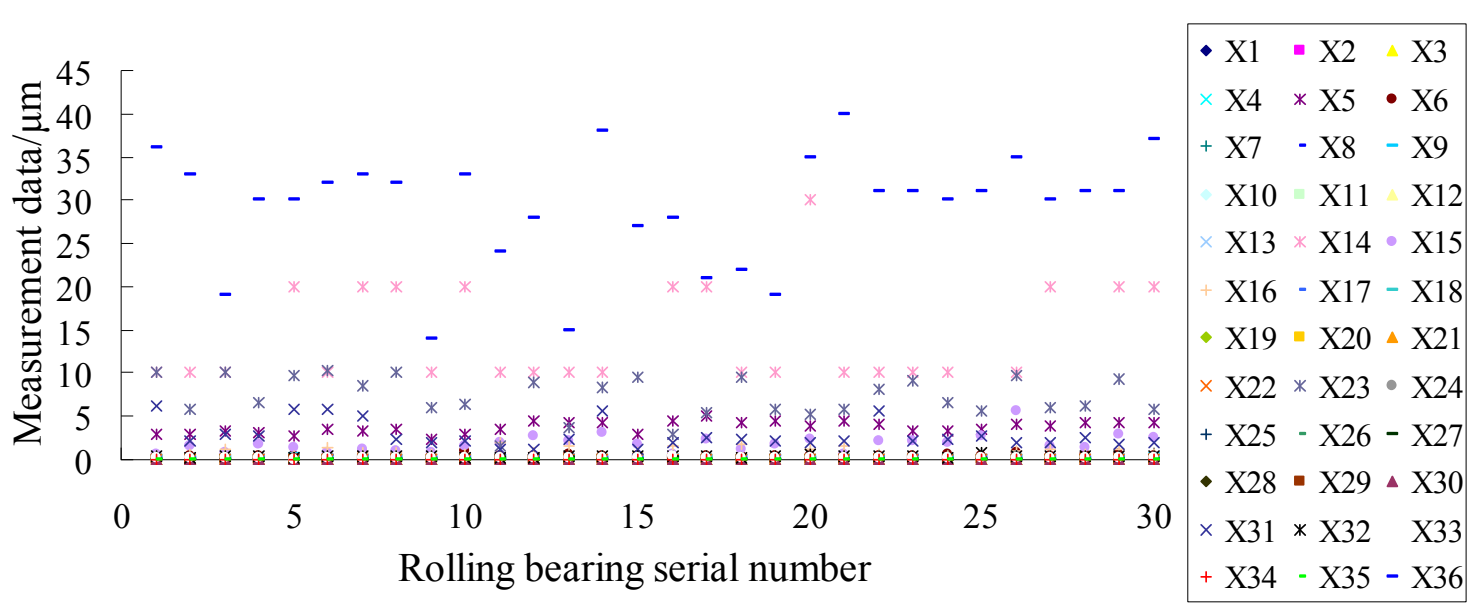

Figure 1. Finite The parameter data of each parameter

\section{Calculation Result}

The correlation degree of each method is sorted to obtain the correlation sequence, and the first ten parameters are taken as the research objects. The results of the correlation sequence of different frequency bands are shown in Table 3, 4 and 5.

Table 3 Influencing factors affecting low frequency vibration velocity

\begin{tabular}{cc}
\hline Method & Top 10 factors \\
\hline Gray correlation degree & $X_{23}, X_{7}, X_{26}, X_{31}, X_{9}, X_{36}, X_{34}, X_{19}, X_{8}, X_{1}$ \\
Absolute correlation degree & $X_{36}, X_{31}, X_{23}, X_{9}, X_{7}, X_{19}, X_{8}, X_{26}, X_{33}, X_{25}$ \\
Relative correlation degree & $X_{12}, X_{23}, X_{31}, X_{34}, X_{19}, X_{7}, X_{9}, X_{36}, X_{26}, X_{8}$ \\
\hline
\end{tabular}

Table 4 Influencing factors affecting intermediate frequency vibration velocity

Method

Gray correlation degree

Absolute correlation degree

Relative correlation degree

Top 10 factors

$X_{26}, X_{7}, X_{9}, X_{23}, X_{8}, X_{36}, X_{19}, X_{34}, X_{31}, X_{25}$

$X_{36}, X_{31}, X_{23}, X_{9}, X_{7}, X_{19}, X_{8}, X_{26}, X_{33}, X_{25}$

$X_{12}, X_{23}, X_{34}, X_{31}, X_{19}, X_{7}, X_{9}, X_{36}, X_{26}, X_{8}$

Table 5 Influencing factors affecting high frequency vibration velocity

\begin{tabular}{cc}
\hline Method & Top 10 factors \\
\hline Gray correlation degree & $X_{26}, X_{8}, X_{9}, X_{25}, X_{36}, X_{3}, X_{7}, X_{33}, X_{23}, X_{24}$ \\
Absolute correlation degree & $X_{36}, X_{31}, X_{23}, X_{9}, X_{7}, X_{19}, X_{8}, X_{26}, X_{33}, X_{25}$ \\
Relative correlation degree & $X_{1}, X_{8}, X_{26}, X_{36}, X_{9}, X_{7}, X_{19}, X_{34}, X_{23}, X_{12}$ \\
\hline
\end{tabular}

\section{Result analysis}

According to the qualitative fusion theory, the main factors affecting the vibration value of three frequency bands can be obtained by fusing the results of the different methods.

Table 6 qualitative fusion results

\begin{tabular}{cc}
\hline Vibration velocity frequency & Influencing factors \\
\hline low frequency & $X_{7}, X_{8}, X_{9}, X_{19}, X_{23}, X_{26}, X_{31}, X_{36}$ \\
intermediate frequency & $X_{7}, X_{8}, X_{9}, X_{19}, X_{23}, X_{26}, X_{31}, X_{36}$ \\
high frequency & $X_{7}, X_{8}, X_{9}, X_{23}, X_{26}, X_{36}$ \\
\hline
\end{tabular}

The main influencing factors of different frequency bands are compared and analyzed. The 
frequency bands and influence degree of the influence factors are shown in Table 7 and 8 .

Table 7 Factors affecting the number of frequency bands

\begin{tabular}{ccc}
\hline Number & Influencing factors & The number of frequency bands \\
\hline 1 & $X_{7}, X_{8}, X_{9}, X_{23}, X_{26}, X_{36}$ & 3 \\
2 & $X_{19}, X_{31}$ & 2 \\
\hline
\end{tabular}

Table 8 The influence degree of main influencing factors on vibration velocity

\begin{tabular}{ccc}
\hline Number & Influencing factors & Degree \\
\hline 1 & inner raceway waviness, inner raceway roughness, roughness (inner & Most \\
2 & flange), convexity (A), roughness (A), clearance & roughness (outer flange), convexity (D)
\end{tabular}

From the table, it can be known that there are 8 main influencing factors, including 3 roller parameters, 3 inner ring parameters, 1 outer ring parameters, and 1 overall parameter. Considering only the quality of the parts, the weight ratio of the influence factors of the roller / inner ring / outer ring on the vibration velocity is $3 / 3 / 1$.It can be seen that the influence of outer ring parameters is less than that of the inner ring parameters

There are 6 most important factors affecting the three frequency bands, including 2 roller parameters, 3 inner ring parameters and 1 overall parameter. Considering only the quality of the parts, the weight ratio of the influence factors of the roller / inner ring on the vibration velocity is $=2 / 3$.

In summary, under the measurement conditions, the biggest influencing factor of the bearing vibration velocity is the inner ring, followed by the roller, and finally the outer ring. This is because in the vibration velocity measurement process, the outer ring of cylindrical roller bearing is fixed, and the rotating shaft is connected to the inner ring. Both the flange of inner and outer rings have an influence on the vibration velocity of the bearing. This is because the main force of the cylindrical roller bearing during the vibration velocity measurement is the axial force, which is mainly conducted by the flange.

\section{Summary}

The results show that for the roller, the roller convexity and roughness average are the weak links of the bearing quality, which shows that the shape, size, quality and roughness of the convexity and the size of roughness have an important influence on the bearing vibration velocity. For rings, the inner ring waviness, roughness and roughness of the inner and outer ring flange are the weak links of the bearing quality. For the overall, the bearing clearance is the weak link of the bearing quality. Because these parameters can be controlled in the production and processing process of bearing, the control of bearing quality during the process of machining and assembly is achievable.

\section{Acknowledgements}

This project is supported by Natural Science Foundation of Henan Province of China (Grant No. 162300410065).

\section{References}

[1] S.S. Creţu and M.I. Benchea: 11th ASME Biennial Conference on Engineering Systems Design and Analysis (Nantes, FRANCE, JUL 02-04, 2012), Vol. 21 (2012), p.565.

[2] T. Lazovic, M. Ristivojevic and R. Mitrovic: Fme Transactions, Vol. 36 (2008) No.3. 
[3] J.V. Poplawski, S.M. Peters and E.V. Zaretsky: Tribology Transactions, Vol. 44 (2001) No.3, p.417.

[4] H. Zoubek, S. Villwock and M. Pacas: Industrial Electronics IEEE Transactions on, Vol. 55 (2008) No.12, p.4270.

[5] A. Leblanc, D. Nelias and C. Defaye: Journal of Sound \& Vibration, Vol. 325 (2008) No.1-2, p. 145 .

[6] K.S. Jiang, G.H. Xu, T.F. Tao and L. Liang: International Journal of Precision Engineering and Manufacturing, Vol. 16 (2015) No.3, p.459.

[7] J.N. Li, W. Chen and Y.B. Xie: 5th World Tribology Congress (Torino, ITALY, SEP 08-13, 2013), Vol. 228, p.1036.

[8] X.T. Xia: Experimental analysis and evaluation of Poor information of rolling bearing (Science Press, China 2007).

[9] Z.Y. Wang: The non-statistical principle with engineering applications. (Science Press, China 2007).

[10]Y.Z. Xu and X.T. Xia: Bearing, (2016) No 8, p.27. (In Chinese) 